\title{
A GRAPHICAL EXTRAPOLATION METHOD TO DETERMINE CANOPY RESISTANCE FROM MEASURED TEMPERATURE AND HUMIDITY PROFILES ABOVE A CROP CANOPY
}

\section{J. CHEN ${ }^{*}$}

Department of Theoretical Production Ecology, Agricultural University, Bornsesteeg 65 , 6708 PD Wageningen (The Netherlands)

(Received January 4, 1984; revision accepted June 29, 1984)

\section{ABSTRACT}

Chen, J., 1985. A graphical extrapolation method to determine canopy resistance from measured temperature and humidity profiles above a crop canopy. Agric. For. Meteorol., 33: $291-297$.

The profile of vapour pressure in Monteith's extrapolation method is replaced by the profile of dew-point temperature. The canopy resistance can then be obtained directly by a graphical extrapolation method from measured temperature and humidity profiles above a crop canopy. The effect of choosing different excess resistances on the canopy resistance thus obtained is discussed.

\section{INTRODUCTION}

For studying the transfer of sensible and latent heat from a crop canopy, the single-layer model (the Penman-Monteith approach) is still widely used because of its simplicity. In this approach a crop canopy is treated as a surface equivalent to a big leaf at a certain level above the ground. In analogy to the stomatal resistance for a single leaf, Monteith (1963) introduced a bulk resistance $r_{\mathrm{c}}$, which is often called canopy resistance, and proposed an extrapolation method to obtain the surface values of temperature and vapour pressure from measured profiles of these two properties above a crop canopy. The value of the canopy resistance can then be calculated.

Experiments have been conducted on various crop canopies to justify the use of $r_{\mathrm{c}}$ as representative of the physiological resistance of the whole canopy. It was found (Monteith, 1981) for barley, sorghum, soybean and sugar beet that the canopy resistance is close to the bulk stomatal resistance of a canopy defined with all the leaf stomatal resistances treated as parallel resistors. That is, the canopy resistance for a crop canopy as a whole plays the same role as the stomatal resistance for a single leaf, as far as the transpiration rate is concerned: the partition of the net radiation absorbed by the whole crop canopy between the sensible and latent heat losses is regulated by the canopy resistance.

\footnotetext{
* Permanent address: Institute of Plant Physiology, Academia, Sinica, 300 Fenglin Road, Shanghai, China.
} 
There has been some discussion (Thom, 1972, 1975; Monteith, 1973) concerning the discrepancy between the locations of the equivalent surface for momentum absorption and for the transfer of sensible and latent heat. This discrepancy is due to a so-called excess resistance to the sensible and latent heat transfer (Thom, 1972). The extrapolation method was improved by including the excess resistance, but the canopy resistance is still obtained by calculation rather than by extrapolation itself. This is because, in Monteith's extrapolation method humidity was specified by the water vapour pressure $e$, hence, two different abscissas for $e$ and the temperature $T$ are required, and the horizontal distance between $T$ and $e$ profiles has no physical meaning.

In the present paper, the water vapour pressure $e$ is replaced by the dewpoint temperature $T d$, so that one abscissa can be used for both temperature and humidity, and the horizontal distance between $T$ and $T d$ profiles now being proportional to the vapour pressure deficit of the air. Moreover, $T$ and $T d$ profiles can be further extrapolated downward, so the canopy resistance can be obtained directly from the graph. Based on this graphical representation, the effect of choosing different values of the excess resistance on the canopy resistance thus obtained can be clearly demonstrated.

\section{MONTEITH'S EXTRAPOLATION METHOD}

Above a crop canopy the windspeed $u$, air temperature $T$ and water vapour pressure $e$ are measured at several heights. These profiles are regarded as logarithmic, so they are represented by straight lines in a graph with $\ln (z-d)$ as ordinate. The parameter $d$ is called zero plane displacement. First, the wind profile is extrapolated downward to intersect the ordinate. The intersection point is $\ln \left(z_{0}\right)$ (Fig. 1 ), where $z_{0}$ is termed the roughness length. This level at the height $d+z_{0}$ is regarded as the location of the equivalent momentum sink of the crop canopy since wind speed and momentum vanish there. Similarly, the profiles of temperature and vapour pressure can be extrapolated to obtain the values of temperature and vapour pressure at some equivalent surface. The problem is: to which height should these profiles be extrapolated?

Monteith (1963) extrapolated these profiles down to the level $\ln \left(z_{0}\right)$, which implies that the location of the equivalent surface for sensible and latent heat sources is assumed the same as that for momentum absorption. The surface values of temperature and vapour pressure thus obtained are denoted by $T(0)_{1}$ and $e(0)_{1}$ in Fig. 1 . There are, however, systematic vertical differences within a crop canopy in the distribution of sources and sinks for heat, water vapour and momentum. It is unlikely that the equivalent surface for sensible and latent heat sources is at the same height as that for momentum. Roughly speaking, compared with the heat and vapour transfer the absorption of momentum is enhanced by pressure forces normal to the leaf surfaces, so the resistance to heat and vapour transfer is higher than that to 


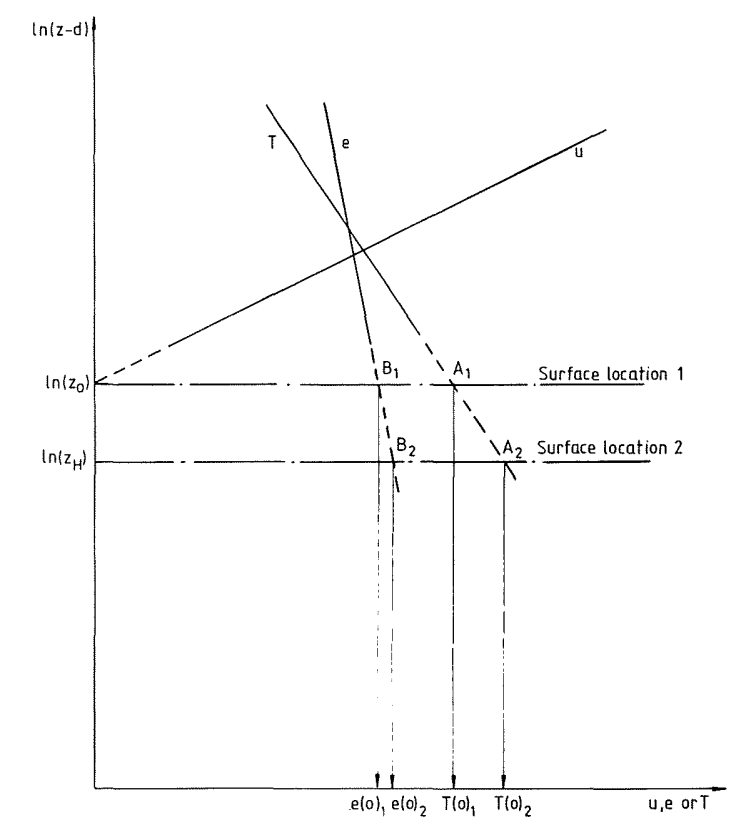

Fig. 1. Monteith's extrapolation method.

momentum transfer. The difference between them is called excess resistance, denoted $r_{\text {ex }}$ (Thom, 1972).

According to the aerodynamic method (Monteith, 1973), the turbulent resistance between two levels $z_{1}$ and $z_{2}, r$, is

$r=\left(1 / k u_{*}\right)\left(\ln \left(z_{2}-d\right)-\ln \left(z_{1}-d\right)\right)$

where $k$ is von Karman's constant, and $u_{*}$ the friction velocity. The parameters $d, z_{0}$ and $u_{*}$ can be obtained from the wind profile (Thom, 1975). Equation 1 means that, in a graph with $\ln (z-d)$ as ordinate, such as Fig. 1, the vertical distance in the figure is in fact a measure of turbulent resistances between relevant levels. The fact that heat and vapour experience an excess resistance compared with momentum means that the equivalent surface for heat and water vapour is below that for momentum, say at $\ln \left(z_{\mathrm{H}}\right)$ shown in Fig. $1, \ln \left(z_{\mathrm{H}}\right)$ being determined by, following eq. 1, the value of the excess resistance

$\ln \left(z_{0}\right)-\ln \left(z_{\mathrm{H}}\right)=k u_{*} r_{\mathrm{ex}}$

To obtain representative surface values of temperature and vapour pressure the profiles of temperature and vapour pressure should be extrapolated to the level $\ln \left(z_{\mathrm{H}}\right)$ as shown in Fig. 1. The intersection points $A_{2}$ and $B_{2}$ represent the surface temperature $T(0)_{2}$ and vapour pressure $e(0)_{2}$, respectively. When $T(0)$ and $e(0)$ are obtained by extrapolation (either as $T(0)_{1}$ and $e(0)_{1}$ or as $T(0)_{2}$ and $\left.e(0)_{2}\right)$, the canopy resistance is calculated from 
$r_{\mathrm{c}}=\left(\rho c_{\mathrm{p}} / \gamma\right)\left(e_{\mathrm{s}}(T(0))-e(0)\right) / \lambda E$

where $\rho c_{\mathrm{p}}$ is the volumetric heat capacity of air, $\gamma$ the psychrometric constant and $\lambda E$ the latent heat flux density above the canopy.

\section{GRAPHICAL EXTRAPOLATION METHOD TO DETERMINE $r_{\mathrm{c}}$}

Besides the water vapour pressure $e$ the dew point temperature $T d$ can also be used to specify the humidity of the air.

The saturated vapour pressure versus temperature curve is more or less exponential in nature, but as a first approximation, a segment of the curve can be replaced by a straight line with a slope $\Delta$, evaluated at a selected temperature $T_{\mathrm{p}}$. Thus,

$T-T d=\left(e_{\mathrm{s}}(T)-e\right) / \Delta$

$e_{\mathrm{s}}(T)=e_{\mathrm{s}}\left(T_{\mathrm{p}}\right)+\Delta\left(T-T_{\mathrm{p}}\right)$

and it follows from these two equations that

$T d=e / \Delta+$ const.

where the constant is equal to $T_{\mathrm{p}}-e_{\mathrm{s}}\left(T_{\mathrm{p}}\right) / \Delta$. Thus, the profile of $T d$ is also logarithmic. The procedure for obtaining the locations of the equivalent surfaces for momentum and heat $\ln \left(z_{0}\right)$ and $\ln \left(z_{\mathrm{H}}\right)$, and the representative surface value of temperature is the same as in Monteith's method. For illustration, the surface location 2 in Fig. 2 is taken as the location for the

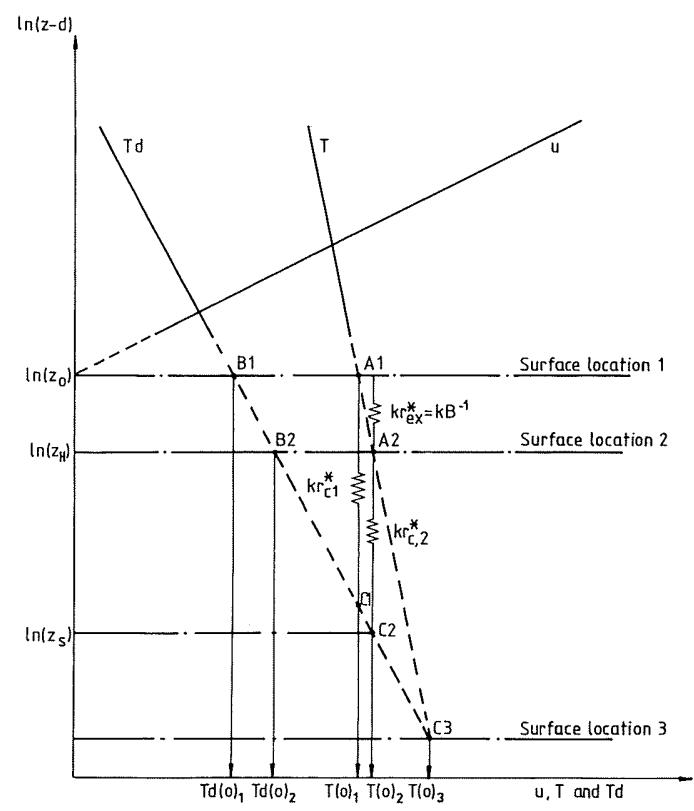

Fig. 2. Extrapolation method determining canopy resistance graphically. 
equivalent surface for heat and vapour. The surface temperature obtained by extrapolation is then determined by the intersection point $A_{2}$ as $T(0)_{2}$. Now extrapolate the profile of dew-point temperature $T d$, to intersect the surface location 2. The intersection point is $B_{2}$ (Fig. 2), which gives the surface value of the dew-point temperature $\operatorname{Td}(0)_{2}$.

The difference between the present method and that of Monteith is that in the former the $T d$ profile can be extrapolated further downward to reach the surface temperature $T(0)_{2}$. The intersection point is denoted by $C_{2}$, and the level is represented by $\ln \left(z_{\mathrm{s}}\right)$. The length of $\mathrm{A}_{2} \mathrm{C}_{2}$ in the graph is proportional to the canopy resistance, which can be proven as follows.

Because the resistances involved have dimension $\mathrm{m}^{-1} \mathrm{~s}$, they can be made dimensionless by multiplying with a velocity. The characteristic velocity scale here is obviously $u_{*}$, so that a dimensionless excess resistance $r_{\mathrm{ex}}^{*}$, which is often denoted $B^{-1}$ and a dimensionless canopy resistance $r_{c}^{*}$ are defined as

$r_{\mathrm{ex}}^{*}=B^{-1}=u_{*} r_{\mathrm{ex}}$

$r_{\mathrm{c}}^{*}=u_{*} r_{\mathrm{c}}$

It follows from eqs. 2 and 7 that the distance between the surface locations 1 and 2 equals $k B^{-1}$, as shown in Fig. 2 .

According to the aerodynamic method, the latent heat flux density is calculated from

$\lambda E=-\left(\rho c_{\mathrm{p}} / \gamma\right) k u_{*}(z-d) \mathrm{d} e / \mathrm{d} z=-\left(\rho c_{\mathrm{p}} / \gamma\right) k u_{*} \mathrm{~d} e / \mathrm{d} \ln (z-d)$

Equation 9 shows that the latent heat flux density is proportional to the slope of the $e$ line in a coordinate system with $\ln (z-d)$ as ordinate. This slope can be replaced, following eq. 6 , by that of the $T d$ line, denoted as $s_{T d}$. Then eq. 9 can be written as

$\lambda E=-k u_{*}\left(\rho c_{\mathrm{p}} / \gamma\right) s_{T d}$

The canopy resistance $r_{\mathrm{c}}$, following eqs. 3,6 and 10 , is now

$r_{\mathrm{c}}=-\left(1 / k u_{*}\right)(T(0)-T d(0)) / s_{T d}$

From Fig. 2 it can be seen that $T(0)-T d(0)$ is the length of $B_{2} A_{2}$, and $-(T(0)-T d(0)) / \mathrm{s}_{T d}$ is the length of $A_{2} C_{2}$. Hence, it has been proven that the length of $A_{2} C_{2}$, which is obtained by extrapolation is proportional to the canopy resistance $r_{\mathrm{c}}$, or more precisely, it equals $k r_{\mathrm{c}}^{*}$, as shown in Fig. 2.

THE EFFECT OF CHOOSING DIFFERENT $r_{\text {ex }}$ ON $r_{c}$

It can be seen from the above argument that discarding Monteith's assumption about the coincidence between the equivalent surfaces for heat and momentum introduced an uncertainty about how to choose the appropriate surface location. Although the excess resistance $r_{\mathrm{ex}}$ and its dimensionless counterpart $B^{-1}$ were introduced, they cannot be determined a priori, or by 
the profiles of wind speed, temperature and vapour pressure above the canopy. In fact, $B^{-1}$ is related to distributions of sinks and sources for momentum, heat and water vapour, so that it can only be simulated by a multi-layer model (Chen, 1984b), or measured experimentally (Chamberlain, 1966). Thom (1972) estimated that $B^{-1}$ is approximately four for several crops. This subject will not be discussed here, since the aim is to examine the effect of choosing different values of excess resistance on the value of the canopy resistance obtained by extrapolation. In fact, this effect can be clearly shown in the graph using the dew-point temperature extrapolation method.

Because the air humidity is specified now by dew-point temperature $T d$ rather than by vapour pressure $e$, the same abscissa for both $T$ and $T d$ can be used. Furthermore, the saturation temperature deficit, $T-T d$, which is proportional to the water vapour deficit, is immediately visible as the horizontal distance between the two lines for $T$ and $T d$.

When the Bowen ratio $(C / \lambda E)$ equals the critical value, $\gamma / \Delta$, the saturation heat flux density $J=C-(\gamma / \Delta) \lambda E$ equals zero. The driving force for $J$, the gradient of the vapour pressure deficit (Chen, 1984a) is then also zero. In this case, the $T d$ line becomes parallel to that of $T$. It can be clearly seen from Fig. 2 that the length of $A_{2} C_{2}$ will be independent of the surface location for heat and vapour when $T$ and $T d$ lines are parallel. In other words, the canopy resistance $r_{\mathrm{c}}$ obtained by extrapolation is independent of the value chosen for the excess resistance, as noted by Thom (1975).

When the $T d$ line deviates from the parallel-to- $T$-line position to the right, as shown in Fig. 2, i.e. $J$ is smaller than zero or the evaporation rate is larger than the so-called equilibrium evaporation rate (Priestley and Taylor, 1972), as the chosen value of the excess resistance increases, the value of the canopy resistance declines. The minimum is zero, shown in Fig. 2 as the surface location 3 . This is the location of an equivalent wet surface giving the same sensible and latent heat flux densities as those above the real canopy. When the $T d$ line deviates to the left, increasing the chosen value of the excess resistance, it increases the canopy resistance. In this case, the equivalent wet surface is not below but above the measured profiles.

It can also be seen from this approach that the larger the deviation of the Bowen ratio from its critical value, the larger the effect of the different values of the excess resistance on the value of the obtained canopy resistance will be. In these cases, it is important to know the value of $B^{-1}$ more accurately in order to obtain a correct value of the canopy resistance.

\section{DISCUSSION}

The linearization of the saturated vapour pressure curve is an approximation; it is feasible only when the linearized region is not too large. This region is determined by the lowest dew-point temperature of the air and the surface temperature (Chen, 1984a). In this paper the saturation vapour 
pressure curve in the whole region is replaced by a single straight line with a slope determined at a selected temperature, usually the mid-point temperature in the region. Because the surface temperature is unknown before extrapolation, iteration is needed. A ten-degree temperature difference is acceptable (Chen, 1984a). When the temperature difference is too large, the temperature interval to be linearized can be split into two parts in order to reduce the error. One represents the region between the lowest dew-point temperature of the air and the dew-point temperature of the surface; the other represents that between the dew-point temperature of the surface and the surface temperature. Then two slopes $\Delta_{1}$ and $\Delta_{2}$ are introduced, evaluated at the mid-point temperature of, respectively, these two regions. It can be shown that eq. 11 for the canopy resistance $r_{\mathbf{c}}$ is modified by a multiplication factor $\Delta_{2} / \Delta_{1}$, while the essential features of the graph do not change.

\section{ACKNOWLEDGMENT}

This work was carried out in connection with a research programme supported by the Ministry of Education and Science of The Netherlands. I gratefully acknowledge this support. I am much indebted to Dr. J. Goudriaan, Prof. C.T. de Wit, Prof. L. Wartena and Prof. J.L. Monteith for many helpful suggestions.

\section{REFERENCES}

Chamberlain, A.C., 1966. Transport of gases to and from grass and grass-like surfaces. Proc. R. Soc. Ser. A, 290: 236-265.

Chen, J., 1984a. Uncoupled multi-layer model for the transfer of sensible and latent heat flux densities from vegetation. Boundary-Layer Meteorol., 28: 213-226.

Chen, J., 1984b. Canopy resistance and excess resistance derived from a multi-layer micrometeorological model. Boundary-Layer Meteorol., submitted.

Monteith, J.L., 1963. Gas exchange in plant communities. In: L.T. Evans (Ed.), Environmental Control of Plant Growth. Academic Press, New York, pp. 95-112.

Monteith, J.L., 1973. Principles of Environmental Physics. Edward Arnold, London.

Monteith, J.L., 1981. Evaporation and surface temperature. Q.J.R. Meteorol. Soc., 107: 1-27.

Priestley, C.H.B. and R.J. Taylor, 1972. On the assessment of surface heat flux and evaporation using large-scale parameters. Mon. Weather Rev., 106: 81-92.

Thom, A.S., 1972. Momentum mass and heat exchange of vegetation. Q. J. R. Meteorol. Soc., 98: 124-134.

Thom, A.S., 1975. Momentum, mass and heat exchange of plant communities. In: J.L. Monteith (Ed.), Vegetation and the Atmosphere, Vol. 1, Academic Press, London, pp. $57-109$. 
